\title{
Tiroiditis crónica de Hashimoto. Serie clínica
}

\section{Hashimoto chronic thyroiditis. Retrospective analysis of 228 patients}

Background: Chronic Hashimoto Thyroiditis (CHT) is the main cause of hypothyroidism. Aim: To report a series of patients with CHT. Material and Methods: Retrospective analysis of a series of 27 men aged $38 \pm 14$ years and 201 women aged $37 \pm 16$ years, evaluated in the private offices of two of the authors. Results: Fifty six percent of patients only had unspecific symptoms at the moment of consultation, $50 \%$ had a family history of thyroid diseases and only $21 \%$ of women had a previous history of goiter. Eighty one percent of patients had clinical or subclinical hypothyroidism, $62 \%$ had both antithyroglobulin and antithyroid peroxidase positive antibodies and $13 \%$ had both antibodies negative. Only $1.4 \%$ of patients had a normal thyroid ultrasound examination. Patients were treated with levothyroxine at a mean dosage of $75 \mu \mathrm{g} /$ day and $53 \%$ achieved an adequate TSH level. Six of ten patients operated due to nodules had a papillary carcinoma. Conclusions: CHT should be sought in the general population, especially those with a family history of thyroid disease. Thyroid ultrasound is seldom normal in patients with CHT. Thyroid substitution should be monitored periodically to achieve adequate TSH levels.

(Rev Med Chile 2010; 138: 827-831).

Key words: Hashimoto disease; Hypothyroidism; Thyrotropin.

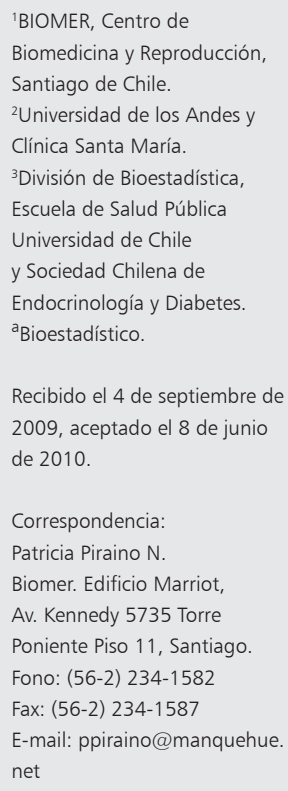

$\tau$ a tiroiditis crónica de Hashimoto (TCH) es una enfermedad autoinmune y constituye la principal causa natural de hipotiroidismo. Fue descrita por primera vez en 1912 por el Dr. Hakaru Hashimoto, quien detectó la presencia de un infiltrado linfocitario que llamó "struma linfomatoso" en la tiroides de estos pacientes ${ }^{1}$. Posteriormente, en 1956 Doniach y Roitt identificaron a la tiroglobulina como el principal autoantígeno que desencadenaba el proceso autoinmune; un año después se encontró un segundo autoantígeno en la fracción microsomal, conocido actualmente como peroxidasa tiroidea ${ }^{2}$.

Hoy en día, el diagnóstico de TCH se hace en relación a la palpación de un tiroides de tamaño normal o aumentado, de consistencia firme y gomosa, indoloro, de bordes netos, superficie irregular; falla funcional tiroidea clínica o subclínica o por la presencia de altos niveles de anticuerpos antitiroideos en sangre (antiperoxidasa, antitiroglobulina o ambos).

Sin embargo, no todos los pacientes con anticuerpos antitiroídeos tienen la enfermedad, estos también se pueden detectar en bajos títulos en pacientes con enfermedad de Basedow Graves clásica, en $20 \%$ de las mujeres sin patología tiroidea ${ }^{3}$ y en $6,6 \%$ de adultos normales, según una publicación de Pineda y cols 4 .

En nuestro país, un estudio realizado en 1992 en Copiapó, comunicó una prevalencia de hipotiroidismo primario en adultos de $8 \%$, con una relación hombre: mujer de 1: $6^{5}$. Los mismos autores detectaron la presencia de anticuerpos antimicrosomales en 13,7\% de la población normal. Estadísticas extranjeras encuentran una prevalencia de $10 \%$ de hipotiroidismo en la población 
femenina y de $2 \%$ en la masculina, apreciándose un aumento de esta enfermedad con la edad ${ }^{6,7}$.

El objetivo de nuestro trabajo fue dar a conocer las características demográficas, clínicas, de laboratorio, ultrasonográficas y de tratamiento de una serie clínica de pacientes evaluados por los autores.

\section{Material y Método}

Se analizó una serie de 228 pacientes. Sus datos provienen de una serie de casos evaluados en forma ambulatoria entre mayo de 2007 y junio de 2008. En gran parte la información obtenida fue retrospectiva, siendo los datos ecográficos prospectivos.

Se analizó la edad del paciente al diagnóstico, historia de bocio en la niñez o adolescencia, antecedentes familiares de enfermedad tiroídea, presencia de otras enfermedades autoinmunes, situación que motivó el diagnóstico, antecedentes de tiroiditis subaguda, niveles hormonales y presencia de anticuerpos antitiroídeos en el momento del diagnóstico y en controles posteriores, características ecográficas y dosis de tratamiento sustitutivo empleado.

Los motivos de consulta fueron: problemas tiroideos en 84 (36,8\%), diabetes mellitus, alteración en el metabolismo glucídico, dislipidemias y obesidad en $54(23,7 \%)$ y otros tales como chequeo médico en 90 (39,5\%).

En relación a los autoanticuerpos se evaluó su presencia considerándose positivo aquellos que estaban aumentados sobre 2 veces el nivel de referencia normal para el laboratorio. En caso de ser positivo, se calculó el número de veces por sobre el valor normal de la técnica utilizada (Quimioluminiscencia y Elisa). El TSH (examen de rutina para las autoras), fue medido por método IRMA, considerándose elevado nieles mayores de $4 \mu \mathrm{U} /$ $\mathrm{ml}$ en 2 mediciones diferentes.

El examen ecográfico fue realizado por 3 radiólogos expertos en ultrasonido tiroideo. Se utilizó un ecógrafo Siemens de alta resolución con transductor lineal de $12 \mathrm{MHz}$, evaluándose la presencia de hipoecogenicidad, heterogeneidad, nódulos o pseudonódulos y aumento de la vascularización al Doppler color.

Se diagnosticó TCH en 228 pacientes que cumplían con a lo menos 2 de las 3 siguientes condiciones: hipotiroidismo clínico o subclínico, presencia de anticuerpos antitiroídeos y alteraciones ecográficas características de esta entidad.

Dado que se analizó una serie de casos, se utilizó mayoritariamente estadística descriptiva, de modo que las variables continuas se describen a través de promedio y desviación estándar si ellas además presentan una distribución simétrica, en caso contrario ellas se describen a través de la mediana y los percentiles 25 y 75 . Las variables categóricas se describen con frecuencias y porcentajes. Se utilizó el test exacto de Fisher para evaluar la asociación entre variables categóricas. Y las variables numéricas fueron comparadas entre dos grupos a través del test t-Student: para muestras independientes y la evolución (en dos instantes) intrapaciente con el test t-Student para muestras pareadas. Dado que la colección de datos es una serie clínica, los test estadísticos sólo fueron usados con el fin de sugerir hipótesis de investigación y no hacer inferencia estadística propiamente tal.

Los datos fueron analizados en el paquete estadístico STATA versión 10.1

\section{Resultados}

En la Tabla 1 se muestran las características demográficas de los pacientes; observándose que $88 \%$ eran mujeres. No hubo diferencias estadísticamente significativas en hombres y mujeres en relación a la edad, momento del diagnóstico ni en los antecedentes familiares de enfermedad tiroidea. En relación a esta última, la más frecuente fue $\mathrm{TCH}$ $(58 \%)$ y luego hipotiroidismo $(15,5 \%)$, este dato fue anamnéstico y probablemente ambas entidades correspondían a lo mismo.

El IMC fue calculado en 221 pacientes (25 hombres y 196 mujeres); el promedio en hombres fue de $27,4 \pm 2,9$ con un rango de 22 a 32,5 y en mujeres fue de $24,7 \pm 4$ con un rango de 17 a 40 . Estos promedios difieren significativamente $(\mathrm{p}=$ 0,0001 ). El $80 \%$ de los hombres y el $44,4 \%$ de las mujeres tuvieron IMC $>$ de 25 . Hubo 3 mujeres con IMC > de 35 (1,53\%), sin embargo, en hombres no se observó obesidad mórbida.

El tiempo de seguimiento de los pacientes fue de 1 mes a 38 años, con mediana de 4 años.

El antecedente de bocio previo sólo estuvo presente en las mujeres (21,6\%); al igual que el antecedente de enfermedad de Graves (4,9\%) y de tiroiditis subaguda (5,7\%). Las enfermedades 
autoinmunes concomitantes más frecuentes fueron, vitiligo y síndrome de Sjögren; sólo 2 hombres tenían otra enfermedad autoinmune, (Diabetes Mellitus tipo 1). El 39,3\% de las mujeres presentaron síntomas inespecíficos; $36,2 \%$ alteraciones de laboratorio y $19,4 \%$ bocio palpable al momento del examen físico inicial. En $3,6 \%$ el diagnóstico se hizo en relación a embarazo, y en una paciente en el estudio de aborto a repetición. En dos mujeres se detectó en la evolución a la cronicidad de tiroiditis postparto.

En los hombres, se hizo el diagnóstico por síntomas inespecíficos en $50 \%$ y por evaluación de laboratorio en la mitad restante (chequeo médico)

En relación a la evaluación de TSH podemos ver que el promedio en hombres y mujeres no tiene diferencia significativa (Tabla 2). Es importante que $81,5 \%$ de nuestros pacientes tuvo TSH al diagnóstico igual o mayor de $4 \mu \mathrm{U} / \mathrm{ml}$; siendo menor de $10 \mathrm{uU} / \mathrm{ml}$ (hipotiroidismo sub clínico) en $42,8 \%$ y con TSH igual o mayor de 10 en $38,7 \%$.

En el momento del diagnóstico los anticuerpos antitiroídeos fueron medidos en 158 pacientes; en 13,3\% fueron ambos negativos y en $62 \%$ ambos positivos (Tabla 3).

Los títulos de anticuerpo antitiroglobulina (Ac anti Tg) al momento del diagnóstico estuvieron elevados en promedio $16,4 \pm 50,3$ veces sobre el valor referido como normal con un rango entre 1,5 a 452 veces. Para los anticuerpos antiperoxidasa tiroidea (Ac anti TPO) el promedio estuvo elevado en 19,1 $\pm 30,2$ veces con un rango entre 1,5 a 251 veces.

Se compararon los promedios de anticuerpos medidos al momento del diagnóstico con aquellos evaluados al mínimo 1 año de seguimiento no encontrándose diferencia significativa entre ambas mediciones (Ac anti Tg:16,6 veces, Ac anti TPO 22,5 veces). Con rango en Ac anti Tg 1,5 a 508 y Ac anti TPO 1,5 a 204.

El estudio ecográfico se hizo en 220 pacientes. Las alteraciones son descritas en la Tabla 4. En sólo 1,4\% no hubo alteraciones.
Tabla 1. Características demográficas

\begin{tabular}{|c|c|c|}
\hline & Hombres & Mujeres \\
\hline Número de pacientes & $27(11,8 \%)$ & $201(88,1 \%)$ \\
\hline Edad del diagnóstico & $38 \pm 14,5$ & $37,3 \pm 15,5$ \\
\hline Bocio previo & $0 \quad(0 \%)$ & $49(21,6 \%)$ \\
\hline $\begin{array}{l}\text { Antecedentes } \\
\text { familiares }\end{array}$ & $14 \quad(52 \%)$ & $116 \quad(58 \%)$ \\
\hline $\begin{array}{l}\text { Antecedentes de enfermedades } \\
\text { autoinmune }\end{array}$ & $2(7,4 \%)$ & $33(16,4 \%)$ \\
\hline $\begin{array}{l}\text { Antecedentes de enfermedad } \\
\text { de Graves }\end{array}$ & $(0 \%)$ & $10 \quad(4,9 \%)$ \\
\hline $\begin{array}{l}\text { Antecedentes de tiroiditis sub } \\
\text { aguda }\end{array}$ & $(0 \%)$ & $13 \quad(5,7 \%)$ \\
\hline
\end{tabular}

Tabla 2. Valor de TSH $[\mu \mathrm{Ul} / \mathrm{ml}]$ al diagnóstico

\begin{tabular}{|lccc|}
\hline & $\begin{array}{c}\text { Hombres } \\
(\mathbf{n = 2 6 )}\end{array}$ & $\begin{array}{c}\text { Mujeres } \\
(\mathbf{n = 1 6 8 )}\end{array}$ & $\begin{array}{c}\text { Total } \\
(\mathbf{n = 1 9 4 )}\end{array}$ \\
\hline Promedio $\pm \mathrm{ds}$ & $17 \pm 19,6$ & $16,9 \pm 27,9$ & $16,9 \pm 26,9$ \\
\hline Rango & $1,01-65$ & $1,04-177$ & $1,01-177$ \\
\hline Mediana & 9,9 & 7,6 & 7,7 \\
\hline
\end{tabular}

Tabla 3. Presencia de anticuerpos antitiroídeos al diagnóstico

\begin{tabular}{|lllcr|}
\hline \multicolumn{4}{c|}{ Ac anti Tg } \\
& & Negativo & Positivo \\
Ac anti & Negativo & $21(13,3 \%)$ & $8(5,1 \%)$ & 29 \\
TPO & Positivo & $31(19,6 \%)$ & $98(62 \%)$ & 129 \\
& & 52 & 106 & 158 \\
\hline
\end{tabular}

Tabla 4. Frecuencia de alteraciones ecográficas sugerentes de TCH

\begin{tabular}{|lcc|}
\hline Alteración & $\mathbf{n}$ & $\mathbf{\%}$ \\
\hline Heterogeneidad & 210 & 95,1 \\
\hline Mayor vascularización & 183 & 82,4 \\
\hline Hipoecogenicidad & 154 & 69,2 \\
\hline Pseudonódulos & 98 & 44,3 \\
\hline Nódulo (s) & 63 & 28 \\
\hline Atrofia & 13 & 5,4 \\
\hline Sin alteraciones & 3 & 1,4 \\
\hline
\end{tabular}


Se diagnosticó nódulos en 63 pacientes; 10 de ellos fueron operados y en 6 la biopsia diferida diagnosticó cáncer papilar. En todos los operados se certificó histológicamente el diagnóstico de $\mathrm{TCH}$. La enfermedad nodular en nuestros pacientes será motivo de otra publicación.

Doscientos diecinueve pacientes se trataron con levotiroxina (LT4), 8 no se trataron y en 1 el dato no estaba consignado. La dosis promedio de LT4 fue de 84,3 $\pm 28,4 \mathrm{ug}$ /día; rango de 25 a 200 ug. La dosis de LT4 usada con mayor frecuencia fue de 75 ug $(33,3 \%)$, luego $100 \mu g(26,6 \%)$ y con 50 $\mu \mathrm{g}(18,4 \%)$. El valor de TSH obtenido durante el tratamiento fue de $2,5 \pm 5,5 \mu \mathrm{U} / \mathrm{ml}$ en promedio, el rango de 0,01 a 72,2 y la mediana de $1,62 \mu \mathrm{U} / \mathrm{ml}$.

En 53,3\% de los pacientes la TSH fue de 0,5 a $2,49 \mu \mathrm{U} / \mathrm{ml}$, y en $30 \%$ de ellos estuvo fuera del rango normal. No hubo correlación entre dosis de LT4 y sexo.

El estudio de correlación entre dosis de LT4 e IMC fue positivo y significativo $(\mathrm{p}=0,01)$; pero en el 53,3\% con adecuada dosis de sustitución (TSH: 0,5 a 2,49 uU/ml) la dosis de LT4 fue independiente del IMC.

En 18 mujeres tuvimos la oportunidad de evaluar la dosis de levotiroxina durante el embarazo, 4 mantuvieron la misma dosis, en 8 fue necesario un incremento de $25 \%$ de la dosis basal y en 6 un incremento de $50 \%$.

\section{Discusión}

A pesar de la alta prevalencia de la TCH encontramos pocas publicaciones en la literatura nacional. La primera de ellas se refiere al análisis de 200 casos de hipotiroidismo primario controlados en 1 año $^{8}$, existe otro trabajo de Michaud $y$ cols, que estudia la prevalencia de TCH en una población joven con bocio'. Por lo anterior, nos pareció interesante comunicar nuestra experiencia con pacientes portadores de esta enfermedad.

En nuestra serie, $88 \%$ fue de sexo femenino lo que da una relación hombre: mujer de 1: 7. Lo comunicado en la literatura mundial varía de 1: 3 hasta 1: $6^{6,7}$. Dentro de los datos anamnésticos es importante destacar la alta frecuencia de antecedentes familiares de TCH e hipotiroidismo; por lo que sugerimos el estudio sistemático de los parientes de primer grado del caso índice.

Respecto al diagnóstico no podemos basarnos sólo en la medición de TSH ya que quedaría un número importante de pacientes no detectados. En nuestra casuística 19\% eran eutiroídeos al momento del diagnóstico; pero presentaban alteraciones ecográficas y presencia de auto anticuerpos en títulos elevados. Dado que en $1 / 3$ de las mujeres y en la mitad de los hombres se hizo el diagnóstico por chequeo con exámenes de laboratorio, es importante solicitar estos exámenes en la evaluación de pacientes mayores de 30 años. El hipotiroidismo subclínico estuvo presente en un porcentaje elevado de nuestra serie, lo que certifica una vez más la importancia de la medición de TSH. Hay $5 \%$ de pacientes que sólo tiene positivo los anticuerpos antitiroglobulina; teniendo otros criterios diagnósticos de TCH presentes. Sin lugar a dudas el anticuerpo más sensible es el Ac anti TPO. La literatura comunica la presencia de anticuerpos antitiroídeos a título bajo en la población general ${ }^{6,7}$ en cifras que fluctúan entre 2 y $16 \%$. Las alteraciones ecográficas descritas en la literatura mencionan casi exclusivamente la hipoecogenici$\operatorname{dad}^{10}$. Hay algunas publicaciones que dudan del beneficio diagnóstico de esta técnica en la $\mathrm{TCH}^{11}$, en nuestra experiencia las alteraciones ecográficas constituyeron el elemento más frecuente y característico de $\mathrm{TCH}$.

Encontramos 6 casos de cáncer papilar, habiéndose operado 10 de un total de 63 pacientes con nódulo(s); (estos datos se comunicarán posteriormente); lo que es mayor que lo comunicado en la población general, en Estados Unidos de Norteamérica, se da una incidencia de $7,7 / 100.000^{12}$. No encontramos linfoma, que sería la neoplasia más frecuente relacionada con $\mathrm{TCH}^{13,14}$. Se sugiere el estudio ecográfico sistemático en los pacientes con $\mathrm{TCH}$ dado su valor diagnóstico y la detección de cáncer en la patología.

Referente al tratamiento, sólo se utilizó levotiroxina. Es importante destacar que a pesar de ser pacientes con alta adhesividad al control hay un elevado porcentaje que no tiene una dosis sustitutiva adecuada. No hubo disminución del título de anticuerpos en el período de seguimiento de los pacientes a pesar del tratamiento con levotiroxina, a diferencia de lo publicado por Schmidt ${ }^{15}$. Llama la atención que hay pacientes que mantienen altos niveles de anticuerpos por períodos prolongados de tiempo; incluso hay coexistencia de hipotiroidismo clínico severo con anticuerpos fuertemente positivos, lo que hace plantearse cuál es el estímulo 
antigénico ya que el parénquima tiroideo noble estaría prácticamente ausente.

Sería importante reevaluar la necesidad de tratamiento en pacientes con $\mathrm{TCH}$ eutiroídeos de manera de tratar farmacológicamente sólo a aquellos que presenten hipotiroidismo clínico y subclínico. Es fundamental en el seguimiento de estos pacientes evaluar por lo menos $1 \mathrm{vez}$ al año el nivel de TSH, el cual debe estar en los rangos normales para así evitar el sobre o sub tratamiento.

En nuestra serie las embarazadas con $\mathrm{TCH}$ necesitaron aumentar la dosis de levotiroxina, por lo que sugerimos medir TSH y T4L precozmente en el embarazo, momento de la gestación en que los niveles de hormona tiroidea de la madre son de especial importancia para el feto

Dada la alta frecuencia, agrupación familiar, posibilidad de tratamiento y constituir un grupo en el cual además se pesquisa cáncer, creemos necesario plantear la importancia de un estudio de prevalencia nacional de la $\mathrm{TCH}$.

\section{Referencias}

1. Lee SL, Odeke S. Hashimoto Thyroiditis. Disponible en www.emedecine.medscape.com 2009 (consultado el 25 de julio de 2009).

2. Portmann L, Hamada N, Heinrich G, DeGroot LJ. Anti-Thyroid peroxidase antibody in patients with autoinmune thyroid disease: possible identity with antimicrosomal antibody. J. Clin Endocrinol Metab 1985; 5: 1001-3.

3. Holowell JG, Staehling NW, Flanders WD, Hannon WH, Gunter EW, Spencer CA, et al. Serum TSH, T4 and thyroid antibodies in the United States population (1988-1994) National Health and Nutrition Examination Survey (NHANES III): J Clin Endocrinol Metab 2002; 2: 489-99.

4. Pineda G, Becker P, Atria A, Aguayo J. Anticuerpos antimicrosomales tiroideos en patología tiroidea: su valor como marcador antigénico. Rev Med Chile 1989; 117: 367-72.

5. Carrasco M, Valenzuela M, Contreras M. Prevalencia de hipotiroidismo primario en Copiapó. Rev Med Chile 1992; 10: 1167-8.

6. Vanderpump MP, Tunbridge WM, French JM, Appleton D, Bates D, Clark F, et al. The incidence of thyroid disorders in the community; a twenty years follow-up of the Whickham Survey. Clin Endocrinol 1995; 1: 55-68.

7. Flynn RW, McDonald TM, Morris AD, Jung RT, Leese GP. The thyroid epidemiology and research study; thyroid dysfunction in the general population. J Clin Endocrinol Metab 2004; 8: 3879-84.

8. Aguayo J, Silva R, Pineda G. Hipotiroidismo primario: análisis de 200 casos controlados en un año. Rev Med Chile 1984; 3: 252-9.

9. Michaud P, Cattani A, Téllez R, Del Valle M, Mahana D. [Chronic Thyroiditis in a young population with goiter]. Rev Med Chile 1988; 1: 24-7.

10. Pedersen OM, Aardal NP, Larssen TB, Varhaug JE, Myking O, Vik Mo H. The value of ultrasonography in predicting autoinmune thyroid disease Thyroid 2000; 3 : 251-9.

11. Nys P, Cordray TP, Merceron RE . Etiologic discussion and clinical relevance of thyroid ultrasonography in clinical hypothyroidism. A retrospective study of 1845 patients. Ann Endocrinol 2009; 1: 59-63.

12. Tuttle RM. Prevalencia de cáncer papilar. Disponible en www.uptodate.com: versión7.2 2009 (consultado el 25 de julio de 2009).

13. Singer JA. Primary Lymphoma of the thyroid. Am Surg 1988; 4: 334-7.

14. Kato I, Tajima K, Suchi T, Aozasa K, Matsuzuka F, Kuma $\mathrm{K}$, et al. Chronic thyroiditis as a risk factor of B-cell lymphoma in the thyroid gland. Jpn J Cancer Res 1985; 16: 1085-90.

15. Schmidt M, Voell M, Rahlff I. Dietlein M, Kobe C, Faust $\mathrm{M}$, et al. Long-term follow-up of antithyroid peroxidase antibodies in patients with chronic autoinmune (Hashimoto thyroiditis) treated with levothyroxine. Thyroid 2008; 7: 755-60. 